\title{
Proposta de transição e continuidade de cuidados da Unidade de Terapia Intensiva
}

\section{para a enfermaria}

\author{
Proposal for the transition and continuity of care from the Intensive Care Unit to the nursing \\ Propuesta de transición y continuidad de la atención de la Unidad de Cuidados Intensivos a la \\ enfermería
}

Louise Bueno Lelli Tominaga

ORCID: https://orcid.org/0000-0002-0367-2617 Universidade Federal do Paraná, Brasil

E-mail: louiselelli@yahoo.com.br

Elizabeth Bernardino

ORCID: https://orcid.org/0000-0003-1321-8562 Universidade Federal do Paraná, Brasil E-mail: elizaber@ufpr.br

Maria Ribeiro Lacerda

ORCID: https://orcid.org/0000-0002-5035-0434 Universidade Federal do Paraná, Brasil

E-mail: mrlacerda55@gmail.com

Maria Manuela Martins

ORCID: https://orcid.org/0000-0003-1527-9940 Escola Superior de Enfermagem do Porto, Portugal

E-mail: mmartins@esenf.pt

Judith Lapierre

ORCID: https://orcid.org/0000-0003-3863-9080 Faculté des Sciences Infirmières, Canadá E-mail: judith.lapierre@fsi.ulaval.ca Otilia Beatriz Maciel da Silva

ORCID: https://orcid.org/0000-0002-1541-5618 Universidade Federal do Paraná, Brasil E-mail: macielotilia2@gmail.com

\begin{abstract}
Resumo
Objetivo: propor elementos para um modelo de transição e continuidade de cuidados dos pacientes da Unidade de Terapia Intensiva (UTI) para a enfermaria de trauma. Método: pesquisa qualitativa, exploratória, realizada com enfermeiros de um hospital de trauma do Sul do Brasil, de maio a junho de 2017, totalizando 22 participantes. Os dados foram coletados por meio de entrevista semiestruturada, com vinheta, e analisados pela Análise de Conteúdo Temático Categorial, usando o software MAXQDA® para organização dos dados. Resultados: emergiram quatro categorias: elementos indicativos para a saída e entrada do paciente em transição da Unidade de Terapia Intensiva (UTI) para a enfermaria; planejamento do processo de transição e continuidade do cuidado do paciente da UTI para a enfermaria; efetivação do processo de transição e continuidade de cuidados do paciente da UTI para a enfermaria; e elementos facilitadores no processo de continuidade de cuidados do paciente em transição da UTI para a enfermaria. O resultado foi um modelo, com os elementos para um plano de transição e continuidade de cuidados dos pacientes da unidade de terapia intensiva para a enfermaria de trauma, centrado no paciente. Conclusão: A criação de práticas profissionais que fomentem o entendimento de transição são estratégias cruciais para a continuidade de cuidados.

Palavras-chave: Cuidado transicional; Continuidade da assistência ao paciente; Alta do paciente; Unidade De Terapia Intensiva.

Abstract

Objective: to propose elements for a model of transition and continuity of care for patients from the Intensive Care Unit (ICU) to the trauma ward. Method: qualitative, exploratory research, carried out with nurses from a trauma hospital in southern Brazil, from May to June 2017, totaling 22 participants. Data were collected through semistructured interviews, with a vignette, and analyzed by Categorical Thematic Content Analysis, using the MAXQDA® software for data organization. Results: four categories emerged: elements indicative of the exit and entry of the patient in transition from the Intensive Care Unit (ICU) to the infirmary; planning the process of transition and continuity of patient care from the ICU to the ward; implementation of the transition process and continuity of care for the patient from the ICU to the ward; and facilitating elements in the process of continuity of care for patients
\end{abstract}


in transition from the ICU to the ward. The result was a patient-centered model, with the elements for a patientcentered plan for the transition and continuity of care for patients from the intensive care unit to the trauma ward. Conclusion: The creation of professional practices that foster an understanding of transition are crucial strategies for the continuity of care.

Keywords: Transitional care; Continuity of patient care; Discharge of the patient; Intensive Care Unit.

\section{Resumen}

Objetivo: proponer elementos para un modelo de transición y continuidad asistencial de los pacientes de la Unidad de Cuidados Intensivos (UCI) a la sala de trauma. Método: investigación cualitativa, exploratoria, realizada con enfermeras de un hospital de trauma en el sur de Brasil, de mayo a junio de 2017, totalizando 22 participantes. Los datos fueron recolectados a través de entrevistas semiestructuradas, con viñeta, y analizados por Análisis de Contenido Temático Categórico, utilizando el software MAXQDA® para la organización de datos. Resultados: surgieron cuatro categorías: elementos indicativos de salida y entrada del paciente en transición de la Unidad de Cuidados Intensivos (UCI) a la enfermería; planificar el proceso de transición y continuidad de la atención al paciente desde la UCI hasta la sala; implementación del proceso de transición y continuidad de la atención del paciente de la UCI a la sala; y elementos facilitadores en el proceso de continuidad asistencial de los pacientes en transición de la UCI a la sala. El resultado fue un modelo centrado en el paciente, con los elementos de un plan centrado en el paciente para la transición y continuidad de la atención de los pacientes de la unidad de cuidados intensivos a la sala de traumatología. Conclusión: La creación de prácticas profesionales que fomenten la comprensión de la transición son estrategias cruciales para la continuidad del cuidado.

Palabras clave: Atención transicional; Continuidad de la atención al paciente; Alta del paciente; Unidad de Terapia Intensiva.

\section{Introdução}

A transição pode ser entendida como um processo determinado por fases de entrada, passagem e saída de uma determinada situação ou por uma mudança significativa na vida de um indivíduo para o qual é exigida uma resposta, após o fato de mudança. Ela antecipa defesas em relação aos riscos à saúde, nos pontos que o indivíduo está mais propenso a atingir picos de vulnerabilidade, modificando-os assertivamente (Meleis, 2010).

Igualmente, inclui todas as passagens no ciclo da vida, mas também decorrentes de condições de saúde que se manifestam, dentro e fora do hospital, incluindo a alta hospitalar e atividades subsequentes a ela, como as necessidades de cuidado Holland \& Harris, 2007; Naylor, Aiken, Kurtzman, Olds \& Hirschman, 2011).

Pacientes internados em Unidades de Terapia Intensiva (UTI) são transferidos para unidades de internação, e viceversa, porque mudam sua condição de saúde e necessitam mobilizar recursos individuais para realizar as adaptações necessárias. A equipe de saúde fornece suporte técnico e emocional para que esta transição se passe da forma mais segura e menos traumática possível (Holland \& Harris, 2007; Naylor et al., 2011).

No entanto, nesse processo de envio e recebimento de pacientes, as transições exigem dos profissionais uniformidade e coordenação dos cuidados, preparação do local e qualidade das informações, e as descontinuidades e as novas hospitalizações são uma realidade. Pacientes de alta da UTI para a enfermaria podem passar por algumas dificuldades, como falta ou insuficiência de orientações dos profissionais de saúde, comunicação deficiente entre unidades, causando insegurança a si e a seus familiares e, principalmente, o temor de retroceder nas condições de saúde, retornando para os a unidade de cuidados intensivos (Oliveira et al., 2021; Petry \& Diniz, 2020).

Alguns autores identificaram determinados domínios que se mostram essenciais para que os cuidados de transição sejam bem-sucedidas: (1) a transferência de informação; (2) a preparação do paciente e do cuidador; (3) o apoio para autogestão, e (4) o empoderamento do paciente (Coleman, Smith, Frank, Eilertsen, Thiare \& Kramer, 2002; Schall, Coleman, Rutherford \& Taylor, 2013).

Justifica-se a realização do presente estudo, considerando que na transição pode haver descontinuidade de cuidados, e que o enfermeiro dispõe de conhecimentos e de posição estratégica para contribuição na diminuição das lacunas desse processo, e fortalecendo a prestação de uma assistência de qualidade. 
Considerando o contexto de transição e continuidade de cuidados delineou-se como questão de pesquisa: Como é o processo de transição de pacientes da UTI para a enfermaria em um hospital de trauma, na perspectiva da continuidade do cuidado, sob a ótica dos enfermeiros? E como objetivo definiu-se: elaborar uma proposta com os elementos necessários para um modelo de transição e continuidade de cuidados dos pacientes da UTI para a enfermaria.

\section{Metodologia}

Pesquisa exploratória de abordagem qualitativa, realizada em duas UTIs e uma unidade geral de um hospital de grande porte, referência no atendimento ao trauma no Sul do Brasil (Polit \& Beck, 2018). Os dados foram produzidos no período de maio a junho de 2017. Os participantes foram 22 enfermeiros que aceitaram participar do estudo de um total de 25 que atuam neste serviço, sendo em número de 17 profissionais das UTIs e 08 da unidade de internação geral. As UTIs do cenário de estudo totalizam 30 leitos, que atendem em grande parte um perfil de paciente vítima de traumas de alta energia, e a unidade de internamento conta com 60 leitos, em expansão, atendendo principalmente pós-operatórios das especialidades de ortopedia, cirurgia geral e neurologia, ambas com atendimento integral subsidiado pelo Sistema Único de Saúde (SUS).

Os critérios de inclusão foram ter vínculo empregatício com o local de estudo e desempenhar atividade assistencial independente do turno de trabalho. Os participantes foram entrevistados pela pesquisadora principal, em seus respectivos locais e horários de trabalho na instituição, tiveram duração em média uma hora, e foram previamente combinadas. Contou-se com o apoio de uma vinheta que apresentava os conceitos de transição e continuidade de cuidados (Polit \& Hungler, 1995). A entrevista tinha um roteiro semiestruturado e foi submetido a dois testes-piloto antes de ser aplicado. Continha questões de caracterização dos participantes, além de outras relacionadas às experiências/vivências do enfermeiro no processo de alta/transição de um paciente da UTI para a enfermaria. Os áudios da entrevista foram gravados e posteriormente transcritos na íntegra pelas pesquisadoras. Todos assinaram o Termo de Consentimento Livre e Esclarecido (TCLE), e os participantes receberam uma codificação representada pela letra "E" maiúscula, para Enfermeiro (a), seguida de numeração de acordo com a ordem das entrevistas, e a letra minúscula "a" para os profissionais da UTI e a letra "b" para os da unidade de internação.

Para a transcrição, sistematização, e análise dos dados, utilizou-se o software MAXQDA ${ }^{\circledR}$ e a Análise de Conteúdo Temático Categorial (ACTC) de Oliveira, sucessivamente ${ }^{8}$. A ACTC é uma técnica composta por nove etapas e procedimentos que apoiam o desenvolvimento da técnica, descritos como: leitura flutuante, intuitiva ou parcialmente orientada do texto; definição de hipóteses; determinação das unidades de registro (URs); marcação no texto do início e final de cada UR observada; definição das unidades de significação (US); e análise temática das unidades de significação (US) (Oliveira, 2016).

O projeto foi aprovado por Comitê de Ética em Pesquisa, CAAE n ${ }^{\circ}$ 1.802.094, parecer consubstanciado número 1.802.094, e seguiu todas as recomendações contidas na Resolução 466/2012 do Conselho Nacional de Saúde, que trata da pesquisa envolvendo seres humanos. Este trabalho faz parte dos estudos de um grupo de pesquisas financiado pelo Edital Universal do Conselho Nacional de Desenvolvimento Científico e Tecnológico, sob o número 406058/2016-4.

\section{Resultados}

Os participantes desta pesquisa tinham em média 35 anos. Eram quinze mulheres e sete homens, com tempo médio de formação de sete anos e meio e tempo médio na instituição de cinco anos e meio, sendo que dezesseis dos enfermeiros tinham experiência prévia no setor de atuação e quinze tinham especialização concluída.

A resposta do participante foi considerada como UR, totalizando 416. Posteriormente a determinação e análise das URs, identificou-se um total de 24 US, e estas foram reagrupadas de acordo com suas semelhanças e promoveram a construção das categorias. As unidades de significação que melhor representaram o cerne de cada categoria estão descritas no Quadro 1. 
Quadro 1 - Nome da categoria, porcentagem da categoria referente às unidades de registro e as unidades de significação correspondentes. Brasil, 2017.

\begin{tabular}{|c|c|c|}
\hline CATEGORIA & $\%$ & UNIDADE DE SIGNIFICAÇÃO \\
\hline \multirow{5}{*}{$\begin{array}{l}\text { Elementos indicativos para a saída da UTI } \\
\text { e entrada do paciente para a enfermaria }\end{array}$} & \multirow{5}{*}{19,38} & Critérios para alta do paciente da UTI para a enfermaria \\
\hline & & $\begin{array}{l}\text { Fatores institucionais envolvidos no processo de transição do } \\
\text { paciente e continuidade de cuidados (UTI) }\end{array}$ \\
\hline & & Fatores clínicos do paciente em transição de cuidados \\
\hline & & $\begin{array}{l}\text { Fatores institucionais envolvidos no processo de transição do } \\
\text { paciente e continuidade de cuidados (enfermaria) }\end{array}$ \\
\hline & & $\begin{array}{l}\text { Preparo para o recebimento do paciente de alta da UTI para a } \\
\text { enfermaria }\end{array}$ \\
\hline \multirow{4}{*}{$\begin{array}{l}\text { Planejamento do processo de transição e } \\
\text { continuidade do cuidado do paciente da } \\
\text { UTI para a enfermaria }\end{array}$} & \multirow{4}{*}{43,87} & Preparo para alta do paciente da UTI para a enfermaria \\
\hline & & Decisão de alta do paciente da UTI para a enfermaria \\
\hline & & $\begin{array}{l}\text { Orientações do enfermeiro no processo de transição do paciente e } \\
\text { continuidade de cuidados }\end{array}$ \\
\hline & & $\begin{array}{l}\text { Papel do enfermeiro na admissão do paciente em transição de } \\
\text { cuidados }\end{array}$ \\
\hline \multirow{3}{*}{$\begin{array}{l}\text { Efetivação do processo de transição e } \\
\text { continuidade de cuidados do paciente da } \\
\text { UTI para a enfermaria }\end{array}$} & \multirow{3}{*}{15,64} & $\begin{array}{l}\text { Tipo de informação necessária para o processo de continuidade de } \\
\text { cuidados }\end{array}$ \\
\hline & & Modo de comunicação no processo de continuidade de cuidados \\
\hline & & Registro de informação no processo de continuidade de cuidados \\
\hline \multirow{5}{*}{$\begin{array}{l}\text { Elementos facilitadores no processo de } \\
\text { continuidade de cuidados do paciente da } \\
\text { UTI para a enfermaria }\end{array}$} & \multirow{5}{*}{26,53} & $\begin{array}{l}\text { Personalização do atendimento ao paciente em transição da UTI para } \\
\text { a enfermaria }\end{array}$ \\
\hline & & $\begin{array}{l}\text { Definição de protocolos no processo de transição do paciente e } \\
\text { continuidade de cuidados }\end{array}$ \\
\hline & & $\begin{array}{l}\text { Preocupação dos enfermeiros com a descontinuidade de cuidados e } \\
\text { lacunas de informação no processo continuidade de cuidados da UTI } \\
\text { para a enfermaria }\end{array}$ \\
\hline & & $\begin{array}{l}\text { Mudanças necessárias para melhora do processo de continuidade de } \\
\text { cuidados da UTI para a enfermaria }\end{array}$ \\
\hline & & $\begin{array}{l}\text { Recursos humanos e materiais envolvidos no processo de transição } \\
\text { do paciente e continuidade de cuidados }\end{array}$ \\
\hline
\end{tabular}

Fonte: Autores (2018).

Da análise de dados do estudo, emergiram quatro categorias intituladas: "Elementos indicativos para a saída da UTI e entrada do paciente na Enfermaria"; "Planejamento do processo de transição e continuidade do cuidado do paciente da UTI para a enfermaria"; "Efetivação do processo de continuidade de cuidados da UTI para a enfermaria"; e "Elementos facilitadores do processo de continuidade de cuidados do paciente em transição da UTI para a enfermaria”. Estas categorias, descritas a seguir, subsidiam a proposta de um modelo que contempla os elementos para um plano de transição e continuidade de cuidados dos pacientes transferidos da UTI para a enfermaria.

\section{Categoria 1: elementos indicativos para a saída da UTI e entrada do paciente na enfermaria}

A categoria 1 representa os detalhes referentes ao processo de alta da UTI para a enfermaria, indicando quais fatores são decisivos para a retirada do paciente da UTI. Ademais, contempla os fatores que estão envolvidos no recebimento do paciente egresso da UTI.

Um dos fatores é que a gente precisa muito da vaga. Tem um paciente que poderia ficar mais tempo na UTI, mas que está melhor do que outros, por exemplo, então a gente acaba trazendo pra liberar a vaga [...] (E1b).

[...] Eu acho que o que impera mesmo é o fator clínico. Melhorou clinicamente, pode ir de alta. Se tiver condições de cuidado ou não, se alguém vai cuidar ou não [...] isso não é levado em consideração. [...] por um lado eu entendo que eu preciso do leito, porque um leito de UTI é para um paciente que está grave. [...] A gente não consegue 
preparar o familiar, mas eu libero o leito para o paciente que está mais grave, e que precisa do cuidado intensivo. [E8a]

\section{Categoria 2: planejamento do processo de transição e continuidade do cuidado do paciente da uti para a enfermaria}

Esta categoria aponta a interface existente entre o preparo do paciente para a alta da UTI, a decisão dessa alta e o modo como o paciente chega à enfermaria. A ênfase, nessas unidades de significação, foi para a atuação do enfermeiro. Emergiram, dessa categoria, as unidades de significação, relacionadas à falta de orientações ao paciente antes de sair da UTI, além de aos fatos de que o preparo para alta na UTI depende da conduta pessoal do profissional e de que esse preparo para alta não é prioridade na UTI. Outros pontos de relevância foram a reflexão sobre a necessidade de preparação do paciente para alta da UTI, da inclusão da família no processo de preparo para alta, com a construção de um vínculo com o enfermeiro, e da participação do enfermeiro na decisão de alta da UTI.

Eu nunca pensei muito nisso. Mas eu acredito que, quando ele sabe que vai sair da UTI, isso gera já um pouco de medo nele, e também acho que há falta de orientação. Às vezes nós falhamos muito nesse aspecto de não orientar muito o doente com relação ao que vai acontecer e a como ele vai se cuidar [...] (E9a).

Ele simplesmente recebe a informação que ele vai de alta. [...] Nós não fazemos este preparo para alta, os profissionais de uma forma geral. (E8a)

É uma conduta mais pessoal nosso mesmo. [...] De orientar. Na equipe tem enfermeiros que esporadicamente não fazem isso, mas eu prefiro fazer. (E1a)

Eu acho que pode ser feito, mas, talvez, na rotina do dia a dia de uma UTI, isso acaba não sendo realizado. (E14a)

Na maioria das vezes a família também vem bem perdida, bem deslocada na situação do quadro do paciente [...] eles não sabem que terão que aprender o procedimento [...] então eles vêm bem perdidos. (E4b)

\section{Categoria 3: efetivação do processo de continuidade de cuidados da UTI para a enfermaria}

Esta categoria foi constituída por questões inerentes à comunicação, como principal ferramenta para efetivação da continuidade dos cuidados: a maneira como a comunicação foi executada, quais as informações pertinentes, e a forma como foram registradas. As unidades de significação que representam a ideia central dessa categoria, apresentadas no Quadro 3, são: o modo de comunicação e que o tipo de informação selecionada depende de uma atitude pessoal; a importância do contato pessoal para troca de informações, sendo que o repasse de informações é insuficiente, há necessidade de melhora dos registros de informações; e por fim a necessidade de um resumo de alta realizado pelo enfermeiro da UTI para ser disponibilizado ao enfermeiro da enfermaria.

[...] eu acho que uma coisa que poderia ser muito eficiente é o registro [...] A gente precisa deixar tudo bem registrado para que outro profissional possa consultar no caso de dúvida ou de precisar de mais informação. (E6a) 
[...] se eu não me satisfaço com o contato telefônico, eu vou pessoalmente à UTI para que essa comunicação seja melhor, e eu visualizo o paciente juntamente com o profissional certo, quando é possível eu procuro fazer sempre assim. (E3b)

Então a gente tem essa dificuldade, em relação à passagem de plantão já melhorou um pouco, mas às vezes tem um pouco de déficit [...]. (E2b)

\section{Categoria 4: elementos facilitadores do processo de continuidade de cuidados do paciente em transição da UTI para a enfermaria}

Nesta categoria, a temática principal esteve relacionada com as mudanças necessárias para melhorias no processo de transição e de continuidade dos cuidados, com a necessidade de colocar o paciente na centralidade de seu cuidado, a adequação de recursos humanos e de materiais para executar uma transição adequada, e a elaboração de protocolos institucionais visando à efetivação da continuidade dos cuidados. As unidades de significação da categoria estão descritas no Quadro 4.

O que eu sei é que muitos pacientes voltam pra UTI, após dois, três dias [...] alguma coisa acontece que eles voltam, e isso é uma falha, isso já é um indicativo de que existe uma falha nesse processo [...]. (E13a)

Para melhorar [...] Não sei se eu estou pensando alto até demais, mas até uma pessoa que pudesse especificamente lidar com isso, só com essas orientações [...] que conseguisse orientar essas famílias e acompanhar melhor lá fora [...] seria um fator interessante [...]. (E9a)

Eu acho mesmo que é a troca de informações entre enfermeiros que melhora o cuidado do paciente. Melhora na troca de informações [...] a conversa é a melhor opção. (E15a)

Quem vai receber esse paciente tinha que ver o paciente antes e pegar esse plantão aqui, na frente do paciente. (E12a)

Criando um protocolo que tenha um documento físico, ou, no caso, um sistema pré-estabelecido que contenham todas as informações que a gente necessite [...]. (E3b)

Sugestões dos elementos para o aprimoramento do processo de transição e continuidade de cuidados da unidade de terapia intensiva para a enfermaria

A proposta de um modelo que contempla os elementos para um plano de transição e continuidade de cuidados dos pacientes da UTI para a enfermaria de trauma, centrado no paciente (Figura 1), foi construída por meio da vivência dos profissionais entrevistados, associado a conceitos encontrados literatura de referência. 
Figura 1 - Processo de transição e continuidade de cuidados da UTI para a enfermaria centrado no paciente.

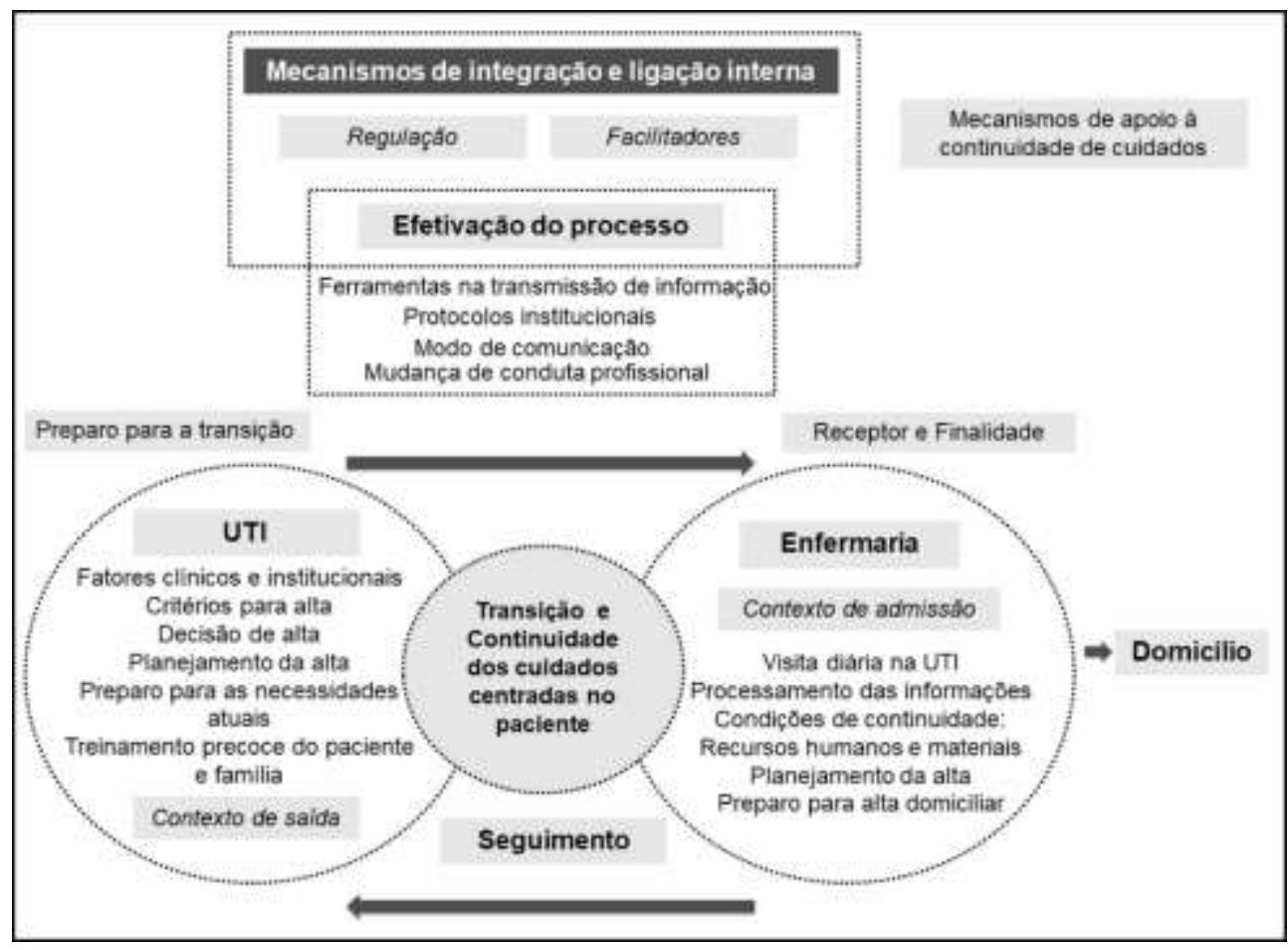

Fonte: Autores (2018).

Para que o processo de transição e continuidade de cuidados centrado no paciente e adequado às suas necessidades seja efetivado, são necessários alguns mecanismos que regulam e facilitam o processo. Esses mecanismos são materializados por meio de ferramentas para a transmissão e registro de informações, da instituição de protocolos dentro do serviço de saúde e também dependem da conduta de cada profissional, seja no preparo para a transição no contexto de saída do paciente, representado pela UTI, ou no preparo para o recebimento do paciente em transição, no contexto de admissão, representado pela enfermaria.

Ainda considerando os mecanismos que integram os diferentes níveis de cuidados e efetivam a ligação entre eles, deve-se considerar principalmente o planejamento da alta, que envolve os critérios para alta do paciente.

\section{Discussão}

Destaca-se que os fatores envolvidos nas altas dos pacientes das UTIs extrapolam as questões de melhora clínica dos pacientes, estando ligados à necessidade de otimização dos leitos e, consequente, à pressão da gestão hospitalar interna e da rede de assistência à saúde. Isso contribui para que o paciente faça transições de forma crítica, e para transições assertivas é necessário o planejamento de cuidados individuais (Germack, Fekieta, Britton, Feder, Rosenberg \& Chaudhry, 2020; Häggström, Asplund \& Kristiansen, 2012; Herve, Zucatti \& Lima, 2020).

A priorização de leitos, pela alta demanda, para pacientes que se encontram em um estado de maior gravidade assistencial geralmente pode ser apressada ou adiada e isso dificulta o planejamento (Balshi et al., 2020; Germack et al., 2020). A falta de um planejamento adequado para a alta pode contribuir para readmissões na UTI, ocasionando aumento da permanência, aumento de custos e maior taxa de mortalidade (Gotur, Masud, Paranilam \& Zimmerman, 2018; Herve, Zucatti \& Lima, 2020; Moreira, Verga, Barbato \& Burghi, 2017). 
Os hospitais têm oportunidades de redução de custos pela adoção das melhores práticas no gerenciamento e planejamento de alta. Para tanto, é necessário envolver procedimentos seguros e estruturados, colaboração, tempo ideal e abordagem multidisciplinar (Brown et al., 2018; Enger \& Andershed, 2018; Rosenberg et al., 2018).

As transições de cuidados bem-sucedidas dependerão da abordagem feita para superação de barreiras para fornecer cuidados centrados no paciente. Isto remete à ideia de que o planejamento da alta é uma parte essencial do processo e, muitas vezes, é negligenciada na UTI (Arora, Sevdalis, Nestel, Woloshynowych, Darzi \& Kneebone, 2010; Cognet \& Coyer, 2014).

Os dados encontrados corroboram com este achado, no sentido de que a orientação sobre os cuidados que o paciente terá em seu próximo nível de cuidados, é essencial, porém, não era realizada. A coordenação entre as unidades de cuidados para transferência dos pacientes, incluindo as informações sobre as necessidades do paciente, reuniões de planejamento de cuidados com famílias e equipes profissionais, deve acontecer precocemente, agregando melhores resultados a processo (Häggström, Asplund \& Kristiansen, 2012; Petry \& Diniz, 2020).

Para a continuidade do cuidado ser uma constante no contexto do planejamento de cuidados, e consequentemente efetivarem-se transições adequadas e seguras, os diferentes profissionais envolvidos neste processo devem adotar estratégias que consolidem o conjunto de práticas que garantam o cuidado integral e coerente ao paciente (Utzumi et al., 2018).

Entre os facilitadores e barreiras para cuidados de qualidade durante a alta hospitalar da UTI, estão uma série de intervenções inter-relacionadas para uma nova engenharia da estrutura e do processo de alta do paciente da UTI. Essas intervenções incluem comunicação profissional-paciente, comunicação profissional-profissional, educação de alta para pacientes e famílias, implementação de melhores práticas, uso de ferramentas para facilitar a alta, educação e treinamento de profissionais nas UTIs e, para um plano de alta ser bem-sucedido, é preciso envolvimento dos profissionais e início precoce das orientações (Grood et al., 2018; Olino et al., 2019).

A criação de um resumo genérico de alta que inclua informações psicossociais, biofísicas e informações adicionais individualizadas para cada paciente, ou seja, uma abordagem centrada no indivíduo para o planejamento de alta, é uma ferramenta de extrema relevância e subsidia o trabalho do enfermeiro (Alves \& Melo, 2019; Blay, Roche, Duffield \& Gallagher, 2017; Psaila, Schmied, Fowler \& Kruske, 2015).

Destaca-se que o processo de transferência é multifacetado, e que utilizar relatórios padronizados é uma forma de facilitar a comunicação, e os discursos confirmam essa necessidade (Blay et al., 2017; Trovo, Cucolo \& Perroca, 2021).

Uma avaliação holística dos pacientes durante a sua estadia hospitalar, identificando suas necessidades específicas, contribui para o desenvolvimento de um documento que trace uma rota entre os níveis de cuidados, e isso é um elemento crucial para a transição. Esse documento deve ser uma combinação de protocolos e sistemas acordados entre profissionais, que inclua as diferentes intervenções a serem realizadas ao longo do processo do paciente, definindo as responsabilidades pela gestão desse paciente em cada ponto de cuidado (Bench, Day \& Griffiths, 2013; Comín-Colet et al., 2016).

Essa prática já é observada em outras realidades fora do cenário nacional, e observa-se que o comparecimento diário na visita multidisciplinar da UTI por enfermeiros de ligação da UTI permite que estes identifiquem em que condições os pacientes vão de alta para uma ala, repercutindo em avanços no processo de tomada de decisão para quando os pacientes podem ter alta (Green \& Edmonds, 2004).

Para uma transição ideal nos cuidados, faz-se necessário um processo para estruturação de um novo design, usando esforços contínuos de melhoria da qualidade e ferramentas efetivas para maximizar a possibilidade de sucesso, sendo a comunicação um componente importante, associada à vontade institucional e a uma equipe com princípios para melhora na qualidade da assistência (Abraham, Burton \& Gordon 2020; Rosenberg et al., 2018). 


\section{Conclusão}

A análise do processo de transição e de continuidade de cuidados dos pacientes da UTI para a enfermaria cumpriu os objetivos do estudo e permitiu a construção de um modelo com os elementos para uma transição ideal, dentro da realidade do contexto analisado, evidenciando que ainda precisamos avançar no entendimento dos processos de transição e da responsabilidade de cada um para seu êxito.

Evidencia-se o papel fundamental e estratégico do enfermeiro durante o planejamento e execução do processo de transição e continuidade de cuidados, devendo assumir o papel de coordenador nesse processo e articulando as ações empregadas com vistas a instrumentalizar o paciente, a família e seus pares.

Entretanto, o estudo evidenciou fragilidades em relação ao conhecimento desses profissionais sobre o tema, o que apresenta uma grande potencialidade de desenvolvimento de educação permanente, discussão clínica e desenvolvimento de outras pesquisas.

Este estudo apresentou limitações quanto ao contexto específico do cenário de sua realização, e UTIs, bem como unidades de internação com outros contextos podem apresentar resultados divergentes em relação à continuidade de cuidados. Tendo em vista os resultados obtidos, para futuras pesquisas sugere-se a realização do estudo em cenários ampliados, e em perspecitvas distintas dos profissionais envolvidos no processo de continuidade de cuidados.

Destaca-se a necessidade de estudos futuros que abordem a temática, para contribuir com o desenvolvimento de estratégias que qualifiquem a transição do cuidado com a elaboração de planos para transição e continuidade de cuidados, com a melhor preparação do paciente e da sua família para o enfrentamento da transição, fomentando o autogerenciamento das suas necessidades de cuidados.

A realização desta pesquisa remete à necessidade de fazer este trabalho em outras perspectivas, ou seja, com os familiares, equipe multidisciplinar e os pacientes, aplicados a diferentes realidades.

O exercício profissional qualificado dos enfermeiros e fundamentado em melhores práticas é essencial para concretização de transições seguras, sendo uma oportunidade para a visibilidade e valorização do trabalho da enfermagem.

\section{Referências}

Abraham J, Burton S \& Gordon HS. (2020). Moving patients from Emergency Department to Medical Intensive Care Unit: tracing barriers and root contributors. Int J Med Inform (133) 104012. https://doi.org/10.1016/j.ijmedinf.2019.104012

Arora, S., Sevdalis, N., Nestel, D., Woloshynowych, M., Darzi, A \& Kneebone, R. (2010). The impact of stress on surgical performance: A systematic review of the literature. Surgery [Internet], 147(3), 318-330.e6. https://www.sciencedirect.com/science/article/pii/S0039606009006175?via\%3Dihub

Alves, M. \& Melo C. L. (2019). Handoff of care in the perspective of the nursing professionals of an emergency unit. Rev Min Enferm, 23:e-1194. http:// www.dx.doi.org/10.5935/1415-2762.20190042

Balshi, A. N et al. (2020). Modified Early Warning Score como preditor de readmissão à unidade de terapia intensiva dentro de 48 horas: um estudo observacional retrospectivo. Revista Brasileira de Terapia Intensiva [online]. (32)2 301-307. https://doi.org/10.5935/0103-507X.20200047. Epub 13 Jul 2020. ISSN 1982-4335. https://doi.org/10.5935/0103-507X.20200047.

Bench, S., Day, T \& Griffiths, P. (2013). Effectiveness of Critical Care Discharge Information in Supporting Early Recovery From Critical Illness. Crit Care Nurse [Internet], 33(3), 41-52. http://www.ncbi.nlm.nih.gov/pubmed/23727851

Blay, N., Roche, M. A., Duffield, C \& Gallagher, R. (2017). Intrahospital transfers and the impact on nursing workload. J Clin Nurs, 26(23- 24):4822-9. https://doi.org/10.1111/jocn.13838

BRASIL. Conselho Nacional de Saúde. Resolução n. 466, de 12 de dezembro de 2012. (2013). Diretrizes e normas regulamentadoras de pesquisa envolvendo seres humanos. Diário Oficial [da] República Federativa do Brasil. Brasília, D.F., Seção 1, p. 59.

Brown, K. N., Leigh, J. P., Kamran, H., Bagshaw, S. M., Fowler, R. A., Dodek, P. M, et al. (2018). Transfers from intensive care unit to hospital ward: a multicentre textual analysis of physician progress notes. Crit Care 22(19) 1-8. doi: 10.1186/s13054-018-1941-0

Coleman, E. A., Smith, J. D., Frank, J. C., Eilertsen, T. B., Thiare, J. N \& Kramer, A. M. (2002). Development and testing of a measure designed to assess the quality-of-care transitions. Int J Integr Care [Internet], 2, e02. http://www.ncbi.nlm.nih.gov/pubmed/16896392 
Comín-Colet, J., Enjuanes, C., Lupó, N. D. J., Cainzos-Achirica, M., Badosa, N \& Verdú, J. M. (2016). Transiciones de cuidados entre insuficiencia cardiaca aguda y cró nica: pasos críticos en el diseñ o de un modelo de atención multidisciplinaria para la prevención de la hospitalizació n recurrente. Rev Esp Cardiol, 69(10), 951-61.

Cognet, S \& Coyer, F. (2014). Discharge practices for the intensive care patient: A qualitative exploration in the general ward setting. Intensive Crit Care Nurs [Internet], 30(5), 292-300. http://www.ncbi.nlm.nih.gov/pubmed/24907890

Enger, R \& Andershed, B. (2018). Nurses' experience of the transfer of ICU patients to general wards: a great responsibility and a huge challenge. J Clin Nurs. (27) 1-2:e186-94. 10.1111/jocn.13911

Germack, H. D., Fekieta, R., Britton, M. C., Feder, S. L., Rosenberg A \& Chaudhry, S. I. (2020). Cooperation and conflict in intra-hospital transfers: a qualitative analysis. Nurs Open 7(2) 634-41. https://doi.org/10.1002/nop2.434

Gotur, D. B., Masud, F., Paranilam, J \& Zimmerman, J. L. (2018). Analysis of Rothman Index Data to Predict Postdischarge Adverse Events in a Medical Intensive Care Unit. J Intensive Care Med. (2) 1-5. 10.1177/0885066618770128.

Green, A \& Edmonds, L. (2004). Bridging the gap between the intensive care unit and general wards-the ICU Liaison Nurse. Intensive Crit Care Nurs [Internet], 20(3), 133-43. http://www.ncbi.nlm.nih.gov/pubmed/15157931

Grood, C., Leigh, J. P., Bagshaw, S. M., Dodek, P. M., Fowler, R. A., Forster A. J, et al. (2018). Patient, family and provider experiences with transfers from intensive care unit to hospital ward: a multicentre qualitative study. CMAJ (190)2 E669-76. https://doi.og/10.1503/cmaj.170588

Hervé, M. E. W., Zucatti, P. B \& Lima, M. A. D. (2020). Transition of care at discharge from the Intensive Care Unit: a scoping review. Revista LatinoAmericana de Enfermagem [online]. (28) e3325. https://doi.org/10.1590/1518-8345.4008.3325. https://doi.org/10.1590/1518-8345.4008.3325.

Häggström, M., Fjellner, C., Öhman, M \& Holmström, M. R. (2018). Ward visits- one essential step in intensive care followup. An interview study with critical care nurses' and ward nurses'. Intensive Crit Care Nurs (49) 21-7. 10.1016/j.iccn.2018.08.011.

Holland, D. E \& Harris, M. R. (2007). Discharge Planning, Transitional Care, Coordination of Care, and Continuity of Care: Clarifying Concepts and Terms from the Hospital Perspective. Home Health Care Serv Q [Internet], 26(4), 3-19. http://www.ncbi.nlm.nih.gov/pubmed/18032197

Meleis, A. I. (2010). Transitions Theory - midle range and situation specific theories in nursing research and practice. Springer Publishing Company. New York: Springer Publishing Company.

Moreira, H. E., Verga, F., Barbato, M \& Burghi, G. (2017). Prognostic impact of the time of admission and discharge from the intensive care unit. Rev Bras Ter Intensiva 29(1) 63-9. 10.5935/0103-507x.20170010

Naylor, M. D., Aiken, L. H., Kurtzman, E. T., Olds, D. M \& Hirschman, K. B. (2011). The Importance of Transitional Care in Achieving Health Reform. Health Aff [Internet], 30(4), 746-54. http://www.healthaffairs.org/doi/10.1377/hlthaff.2011.0041

Olino, L., Gonçalves, A. C., Strada, J. K. R., Vieira, L. B., Machado, M. L. P., Molina, K. L, et al. (2019). Effective communication for patient safety: transfer note and Modified Early Warning Score Rev Gaúcha Enferm, (40)esp e20180341. https://doi. org/10.1590/1983-1447.2019.2018034.

Oliveira, D. C de. (2016). Análise de conteúdo temático-categorial: uma técnica maior nas pesquisas qualitativas. In: Lacerda MR, Costenaro RGS, organizadores. Metodologias da Pesquisa para a Enfermagem e Saúde: da teoria à prática.

Oliveira, L. S. et al. (2021). Práticas de enfermeiros de um hospital universitário na continuidade do cuidado para a atenção primária. Escola Anna Nery, (25)5 e20200530. https://doi.org/10.1590/2177-9465-EAN-2020-0530.

Petry, L \& Diniz, M. B. C. (2020). Communication between teams and the care transfer of critical patients. Rev Rene. (21) e43080. https://doi. org/10.15253/2175-6783.20202143080.

Polit, D. F \& Beck, C. T. (2018). Fundamentos de Pesquisa em Enfermagem: Avaliação de Evidências para a Prática da Enfermagem. Brasil: Editora: Artmed. $9^{\mathrm{a}}$ ed.

Polit, D. F \& Hungler, B. P. (1995). Fundamentos de pesquisa em enfermagem. Artes Médicas. (3a ed.).

Rosenberg, A., Britton, M. C., Feder, S., Minges, K., Hodson, B., Chaudhry, S. I, et al. (2018). A taxonomy and cultural analysis of intra-hospital patient transfers. Res Nurs Health (41)4 378-88. https://doi.org/10.1002/nur.21875

Schall, M., Coleman, E., Rutherford, P \& Taylor, J. (2013). How-to Guide: Improving Transitions from the Hospital to the Clinical Office Practice to Reduce Avoidable Rehospitalizations [Internet]. Institute for Healthcare Improvement. Cambridge: Institute for Healthcare Improvement. Recuperado em: http://www.ihi.org/resources/Pages/Tools/HowtoGuideImprovingTransitionsHospitaltoOfficePracticeReduceRehospitalizations.aspx

Trovó, S. A., Cucolo, D. F \& Perroca, M. G. (2021). Transfer of patients in hospital units: impacts on nursing workload. Revista da Escola de Enfermagem da USP [online]. (55) e0327. https://doi.org/10.1590/S1980-220X2020024903727. https://doi.org/10.1590/S1980-220X2020024903727.

Utzumi, F. C., Catafesta, L. M. R., Bernardino, E., Gomes, I. M., Aued, G. K \& Sousa, S. M. de. (2018). Continuidade do cuidado e o interacionismo simbólico: um entendimento possível. Texto \& Contexto - Enfermagem, 27(2), e4250016. https://dx.doi.org/10.1590/0104-070720180004250016 